University of New Hampshire

University of New Hampshire Scholars' Repository

Space Science Center

Institute for the Study of Earth, Oceans, and

Space (EOS)

1994

\title{
COMPTEL upper limits for Seyfert galaxies
}

\author{
M Maisack \\ University of Tubingen \\ W Collmar \\ Max-Planck-Institut für extraterrestriche Physik \\ G G. Lichti \\ Max-Planck-Institut für extraterrestrische Physik \\ V Schonfelder \\ Max-Planck-Institut für extraterrestrische Physik \\ H Steinle \\ Max-Planck-Institut für extraterrestriche Physik
}

See next page for additional authors

Follow this and additional works at: https://scholars.unh.edu/ssc

Part of the Astrophysics and Astronomy Commons

\section{Recommended Citation}

COMPTEL upper limits for Seyfert galaxies Maisack, M. and Collmar, W. and Lichti, G. G. and Schönfelder, V. and Steinle, H. and Bloemen, H. and Hermsen, W. and McConnell, M. and Stacy, G. and Barr, P. and Williams, O. R., AIP Conference Proceedings, 304, 556-560 (1994), DOI:http://dx.doi.org/10.1063/1.45652

This Conference Proceeding is brought to you for free and open access by the Institute for the Study of Earth, Oceans, and Space (EOS) at University of New Hampshire Scholars' Repository. It has been accepted for inclusion in Space Science Center by an authorized administrator of University of New Hampshire Scholars' Repository. For more information, please contact Scholarly.Communication@unh.edu. 


\section{Authors}

M Maisack, W Collmar, G G. Lichti, V Schonfelder, H Steinle, H Bloemen, W Hermsen, Mark L. McConnell, G Stacy, P Barr, and O R. Williams 


\section{AIP $\mid$ proceedings}

\section{COMPTEL upper limits for Seyfert galaxies}

M. Maisack, W. Collmar, G. G. Lichti, V. Schönfelder, H. Steinle, H. Bloemen, W. Hermsen, M. McConnell, G. Stacy, P. Barr, and O. R. Williams

Citation: AIP Conference Proceedings 304, 556 (1994); doi: 10.1063/1.45652

View online: http://dx.doi.org/10.1063/1.45652

View Table of Contents:

http://scitation.aip.org/content/aip/proceeding/aipcp/304?ver=pdfcov

Published by the AIP Publishing

\section{Articles you may be interested in}

Comptel gammaray observations of the quasars CTA 102 and 3C 454.3

AIP Conf. Proc. 304, 644 (1994); 10.1063/1.45665

EGRET observations of Mrk 421 in Phase 1 and Phase 2 of the Compton Observatory's viewing program-A summary

AIP Conf. Proc. 304, 582 (1994); 10.1063/1.45656

The origin of Xrays and gammarays from Seyferts galaxies

AIP Conf. Proc. 304, 525 (1994); 10.1063/1.45647

The canonical Seyfert spectrum: The implications of OSSE observations AIP Conf. Proc. 304, 515 (1994); 10.1063/1.45646

COMPTEL detection of the variable radio source GT $0236+610$ AIP Conf. Proc. 304, 324 (1994); 10.1063/1.45581 


\title{
COMPTEL UPPER LIMITS FOR SEYFERT GALAXIES
}

\author{
M. Maisack \\ Astronomisches Institut der Universität Tübingen, 72076 Tübingen, Germany \\ W. Collmar, G.G. Lichti, V. Schönfelder, H. Steinle \\ Max-Planck-Institut für extraterrestrische Physik, 85740 Garching, Germany \\ H. Bloemen, W. Hermsen \\ SRON-Leiden, P.O. Box 9504, 2300 RA Leiden, The Netherlands \\ M. McConnell, G. Stacy \\ University of New Hampshire, Durham NH 03824, USA \\ P. Barr, O.R. Williams \\ Astrophysics Division, ESTEC, 2200 AG Nordwijk, The Netherlands
}

\begin{abstract}
The gamma-ray emission of Seyfert galaxies has fallen far short of pre-GRO expectations. No single object of this class has been detected by either COMPTEL or EGRET, and OSSE has detected only a fraction of the Seyferts expected. To derive a more stringent upper limit to the emission from these objects in the energy ranges 0.75 to 1 and 1 to $3 \mathrm{MeV}$, we have summed a large number of COMPTEL observations acquired during Phase 1 of the GRO mission. From a total of 47 observations of 23 individual $X$-ray selected Seyfert galaxies, we derive preliminary upper limits of $8 \times 10^{-8}$ photons / $\left(\mathrm{cm}^{2} \mathrm{~s} \mathrm{keV}\right)$ in the $0.75-1 \mathrm{MeV}$ band and $1 \times 10^{-8}$ photons $/\left(\mathrm{cm}^{2} \mathrm{~s} \mathrm{keV}\right)$ in the 1-3 $\mathrm{MeV}$ band.
\end{abstract}

\section{OBJECTIVE OF THIS STUDY}

Observations of Seyfert galaxies between 10 and $100 \mathrm{keV}$ have shown that emission above $10 \mathrm{keV}$ is common. Balloon observations also detected $\mathrm{MeV}$ emission from NGC 4151 and MCG 8-11-11 (Perotti et al. 1981). Spectral analysis has shown that the $\mathrm{X}$-ray spectra of Seyferts, in general, can be described by a "canonical" power law shape with photon index $\alpha=1.7$ (Rothschild et al. 1983, Turner 
and Pounds 1989). Quasars, on average, have steeper X-ray spectra (Williams et al. 1992). Assuming that this spectral shape extends up to $\mathrm{MeV}$ energies, it was expected that COMPTEL ${ }^{1}$ would be able to detect the brightest Seyferts (e.g. v.Montigny et al. 1989).

The picture has changed dramatically, though, since the launch of GRO. OSSE has performed more than twenty pointed observations of Seyfert galaxies, and those that were detected have shown steep spectra which do not extend beyond $511 \mathrm{keV}$ (Cameron et al. 1993, Maisack et al. 1993). Johnson et al. (1993) find a thermal spectrum from the accumulated data of a sample of Seyfert galaxies observed by OSSE (excluding the 4 brightest objects) which can be described by a spectrum that has an e-folding energy of $\approx 50 \mathrm{keV}$. Neither COMPTEL nor EGRET have detected a single Seyfert galaxy, in marked contrast to the detection of a number of blazars.

To date, no upper limits at $\mathrm{MeV}$ energies for a large sample of Seyfert galaxies have been derived, but a similiar study has recently been undertaken by Maisack, Wood and Gruber (1993) based on data of the HEAO-A4 all sky survey of active galaxies. In this study of AGN not detected as individual sources in the survey, only a sample of Seyfert 1 galaxies (but not quasars or BL Lacs) yielded a significant signal at energies up to $100 \mathrm{keV}$.

In the study presented here we derive limits on the average emission from a large sample of Seyfert galaxies. This method improves on the sensitivity of single COMPTEL observations of individual sources, and will provide more stringent limits on the MeV emission of Seyferts as a class.

\begin{tabular}{|cc|}
\hline \multicolumn{2}{|c|}{ Table I: Source List } \\
\hline NGC 2992 & NGC 3783 \\
NGC 4051 & NGC 4151 \\
NGC 4593 & NGC 5506 \\
NGC 5548 & NGC 6814 \\
NGC 7314 & NGC 7469 \\
NGC 7582 & MCG -2-58-22 \\
MCG -6-30-15 & MCG 8-11-11 \\
3C 111 & 3C 120 \\
IC 4329A & Mkn 335 \\
Mkn 509 & Mkn 841 \\
ESO 141-55 & III Zw 2 \\
Akn 120 & \\
\hline
\end{tabular}

\footnotetext{
${ }^{1}$ for a detailed description of the COMPTEL instrument, see Schönfelder et al. 1993, ApJS, 86,637
} 


\section{SOURCE SELECTION CRITERIA}

For this study we have selected a sample of 23 Seyfert Galaxies according to the following criteria.

- detection by HEAO-A4 (Rothschild et al. 1983)

- hard spectra and high emission level during EXOSAT observations (Turner and Pounds 1989)

- observations by OSSE (some simultaneous)

\section{DATA ANALYSIS}

As a first step, we have performed an analysis using the binned event, exposure and geometry files (the so called DRE / DRX / DRG datasets), generated as part of the routine analysis of the Phase 1 COMPTEL observations. For every observation when one of our candidate sources was located within 25 degrees of the COMPTEL $\mathrm{Z}$-axis (where the effective area is $>50 \%$ ), we have carried out a maximum likelihood analysis (using the SRCLIX ${ }^{2}$ program, Bloemen et al. 1993) to produce likelihood ratio, count, and flux maps covering a 10 by 10 degree region centered on the respective Seyfert position. Since some of the sources were observed more than once, a total of 47 sets of maps could be generated for each energy interval (0.75-1 MeV, 1-3 MeV). Finally the resulting likelihood, count and flux matrices were added (and errors propagated) to test for a significant excess of counts in the centre of the summed maps.

An improved method of analysis involves the superposition of the binned threedimensional event matrices (DRE datasets) produced from the processed event files (EVP). This superposition is achieved by first producing a DRE dataset with the reference system centered on the respective Seyfert galaxy. The resulting DRE, geometry and exposure files are then added (by using SKYADR ${ }^{2}$ ) and treated as a single long observation of a single source. This set of summed datasets is then again analysed by SRCLIX ${ }^{2}$ to derive likelihood, flux and errors in a 10 by 10 degree field surrounding the candidate Seyfert. This work is currently in progress.

\section{RESULTS}

None of the observations analysed has shown significant flux from a single Seyfert, nor has our preliminary analysis of the data shown a significant signal from our

\footnotetext{
${ }^{2}$ all programs used in this project belong to the standard COMPTEL analysis software COMPASS. For a description of COMPTEL data analysis methods, see Schönfelder et al. 1993 and references therein. Data analysis was performed at MPE in Garching.
} 
cumulative dataset of summed observations. We have derived preliminary, conservative upper limits on the average emission from Seyfert galaxies, which will be improved after further analysis.

The upper limits $(3 \sigma)$ we derive are

$8 \times 10^{-8}$ photons / $\left(\mathrm{cm}^{2} \mathrm{~s} \mathrm{keV}\right)$ in the $0.75-1 \mathrm{MeV}$ band and

$1 \times 10^{-8}$ photons $/\left(\mathrm{cm}^{2} \mathrm{~s} \mathrm{keV}\right)$ in the $1-3 \mathrm{MeV}$ band

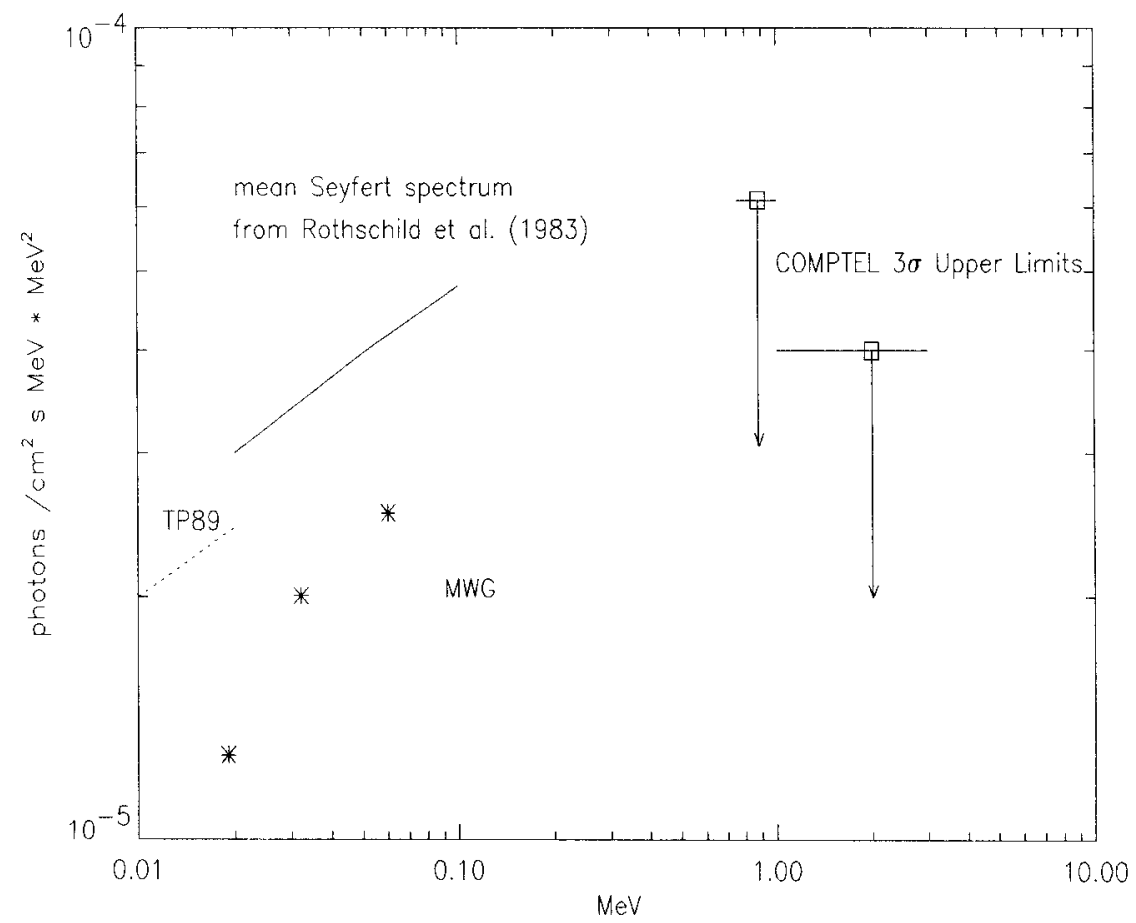

Figure 1: COMPTEL Upper Limits Compared to Results From Previous Samples

Here, we have not considered possible variability of the sources in our arguments. We find that the distribution of likelihood, count and flux values in the summed 10 by 10 degree field we get by either method is not flat, although there should be no other sources in it that are known to emit strongly at $\mathrm{MeV}$ energies. To verify this, we have scanned the HEAO-A4 and A2 Piccinotti catalogs in the HEASARC database ${ }^{3}$ for possible contaminating sources. None were found, except when two Seyferts of our sample are closer to each other than $10^{\circ}$. Therefore, the deviations from a flat field must be due to systematic effects, caused by e.g. background

\footnotetext{
${ }^{3}$ The HEASARC database is operated by NASA - Goddard Space Flight Center
} 
subtraction residuals. Nevertheless, we estimate the systematic uncertainties to be much smaller than the statistical uncertainties. a detailed evaluation is in progress.

The upper limits reported here fall below the extrapolation of "canonical" power law spectra with photon index $\alpha=1.7$ observed in the X-ray band for those Seyfert galaxies used in this study. This is true for the sample studied by Turner and Pounds (1989, EXOSAT data), Rothschild et al. (1983, A4 pointed data) and Maisack, Wood and Gruber (1993, A4 survey data), as can be seen in Fig. 1. If one assumes that the canonical index is steeper, e.g. $\alpha \approx 2.0$, as recently suggested (e.g. Zdziarski et al. 1990), the COMPTEL upper limits are consistent with an extrapolation of the low-energy spectrum of Seyfert galaxies. However, OSSE observations indicate that the average spectrum of Seyfert galaxies is steeper than $\alpha=2$ above $50 \mathrm{keV}$.

\section{SUMMARY}

By studying a large sample of Seyfert galaxies, we find that these objects are not prominent emitters at $\mathrm{MeV}$ energies, contrary to pre-GRO expectations. By superposition of the COMPTEL dataspaces of a large number of observations of Seyfert galaxies, we have derived a more stringent upper limit on the emission of these objects at $\mathrm{MeV}$ energies. We find that the average photon index of Seyfert galaxies between $10 \mathrm{keV}$ and $1 \mathrm{MeV}$ must be greater than the canonical value of Rothschild et al. (1983) of $\alpha=1.7$.

MM acknowledges support by DARA grant 50 OR 92054 .

\section{REFERENCES}

Bloemen, H. et al. 1993. ApJS, in press

Cameron, R.A. et al. 1993, in: Proceedings of the 1st Compton Symposium, AIP Conf. Proc. 280, p.461

Johnson, W.N. et al. 1993. these proceedings

Maisack, M. et al. 1993, ApJ 407, L61

Maisack, M., Wood, K.S. and Gruber, D.E. 1993, in: Proceedings of the 1st Compton Symposium, AIP Conf. Proc. 280, p.528

Perotti, F. et al. 1981. ApJ 247, L63

Rothschild, R.E. et al. 1983, ApJ 269, 423

Schönfelder, V. et al. 1993, ApJS, 86, 637

Turner, T.J. and Pounds, K.A. 1989, MNRAS 240, 833

v.Montigny, C. et al. 1989, 1st GRO Workshop, 4-30

Williams, O.R. et al. 1993, ApJ, 389, 157

Zdziarski, A.A. et al. 1990, APJL, 363, L1 